\title{
BUILDING A REDUCED DICTIONARY OF RELEVANT PERFUSION PATTERNS FROM CEUS DATA FOR THE CLASSIFICATION OF TESTIS LESIONS
}

\author{
Tommaso Favaron ${ }^{1}$, Dean Y. Huang ${ }^{2}$, Kirsten Christensen-Jeffries ${ }^{3}$, \\ Robert E. Eckersley, Paul S. Sidhu ${ }^{2}$, Enrico Grisan ${ }^{1,3}$ \\ ${ }^{1}$ Department of Information Engineering, University of Padova, Italy \\ ${ }^{2}$ Department of Clinical Radiology, King's College Hospital, London, UK \\ ${ }^{3}$ Department of Imaging Science and Biomedical Engineering, King's College London, UK
}

\begin{abstract}
Radical orchifunicolectomy has traditionally been the main clinical treatment for small testicular masses (STMs); however STMs represent a constantly increasing and often incidental finding. Since many of them result benign, a more conservative testis-sparing surgery was proposed, but it requires a preliminary differentiation between benign and malignant masses: this however remains challenging.

Although common understanding in radiology and oncology is that perfusion patterns might provide a useful information about the type of masses, no guidelines or consensus is available for the differentiation of STMs.

We propose to build a dictionary of relevant perfusion patterns, extracted using non-negative matrix factorization on pixel-wise time-intensity curves from contrast-enhanced ultrasound data.

When data from a lesion are reconstructed using this dictionary, a vector containing the frequency of utilization of each pattern can be used as a tissue signature.

Using this signature, a support vector machine classifier has been trained, and the cross validated accuracy reached $100 \%$ in our pilot cohort.
\end{abstract}

Index Terms - CEUS, ultrasound non-negative matrix factorization, dictionary learning, SVM, cancer

\section{INTRODUCTION}

Testicular tumours has been increasing for many decades. Despite its relatively low incidence, they contribute to approximately $1 \%$ of all malignancies in males, they appear at a young age adversely affecting the quality of life of patients. The majority of testicular tumours arise from spermatogenic cells (germ cells) and are almost uniformly malignant. Non-germ cell tumours represent the remainder of primary and secondary testicular tumours, and they consist of sex cord stromal tumours (Leydig or Sertoli cell tumours), lymphoma and metastasis [1]; in particular Leydig cell tumours (LCT) are benign and are elective for testis-sparing surgery.
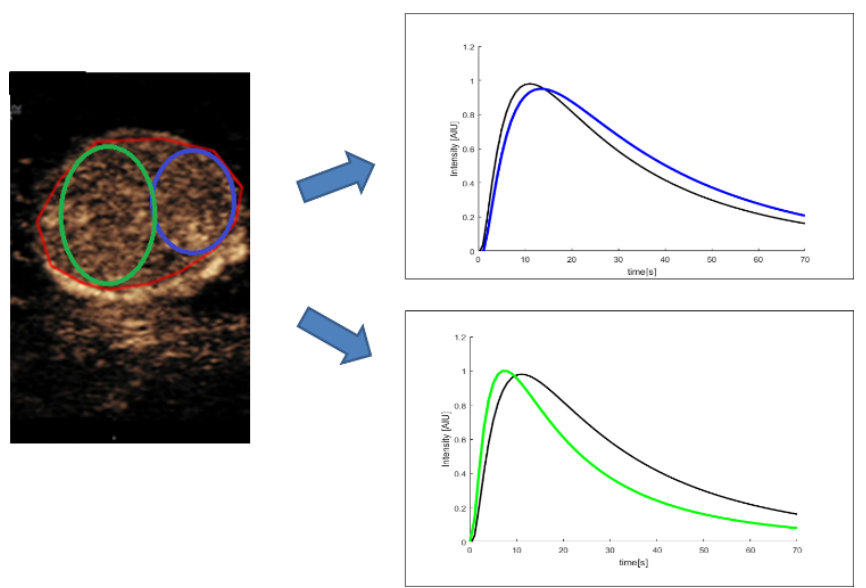

Figure 1 Effects of tissue heterogeneity. The perfusion parameters extracted from the average TIC (black line, right panels) of a region of interest (red outline, left panel), can be markedly different from those estimated on TICs extracted from sub-regions (green and blue lines).

Ultrasound is the first-line imaging technique for many testicular diseases, being a sensitive and accurate technique for the detection of testicular abnormalities. Additionally, ultrasound is the one scrotal imaging technique that a patient will undergo prior to surgery. However, even if B-mode ultrasound proves extremely sensitive for the detection of testicular masses, it does not provide the possibilities of differentiating the tumours type, i.e. histological diagnosis, which could potentially result in a conservative surgical approach [2]. Currently, there are no ultrasound criteria that allow definitive differentiation of benign from malignant testicular lesions [1], so that the diagnosis can only be carried out with an histological examination. Unfortunately, at variance with many cancers, where a biopsy can be obtained, the only way to examine a testicular lump is by removing the affected testicle completely [3].

One of the most important parameters for assessing the tumor status of a particular tissue is the degree of vascularization, since it reflects the metabolic requirements of the surrounding tissue, characterizes tissue pathology, and impacts the effectiveness of the therapy [4]. The macroscopic 


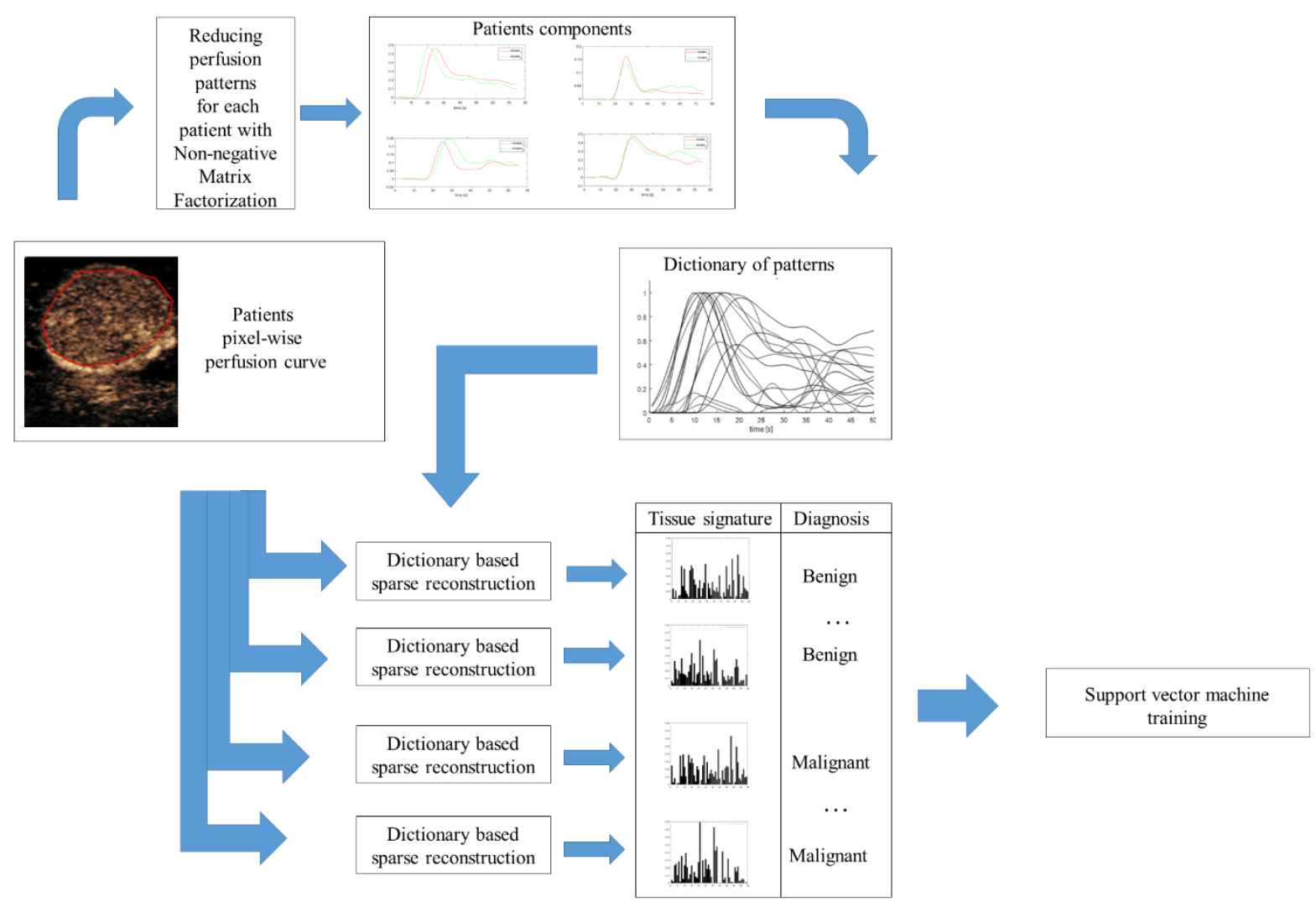

Figure 2 Supervised training phase. From each patient, pixel-wise perfusion curves are extracted and decomposed with NMF into a fixed number of components. All components from all patients create a dictionary that is used to sparsely reconstruct the pixel-wise perfusion curves, obtaining the perfusion signature for each patient. This is used as feature input to train a SVM classifier.

characteristics of tumor microvasculature are very different from those of a normal one [5], that is reflected in an altered and heterogeneous blood flow. [6].

Contrast-enhanced ultrasound (CEUS) has become an important part of clinical radiography to observe blood perfusion in organs and measure blood flow rate. It is obtained by imaging an ultrasound contrast agent composed by microbubbles whose size constrain them to remain confined purely intravascular. Traditionally, a set of parameters are extracted from the CEUS data to quantify the perfusion patterns [7] [8] [9], and they have been proposed as biomarkers of therapeutic response in human cancers [10] [11]. However, even if has been reported that the use of CEUS improves the characterization of testicular lesions, through the qualitative evaluation of vascular flow [12], the use of CEUS-derived parameters showed limited success in differentiating testicular masses [13].

At variance with other data-driven approaches where overcomplete dictionaries of patterns are learnt [14], we propose to build a dictionary using a limited set of patterns estimated by means of Non-negative Matrix Factorization (NMF). The identification of the patterns in the dictionary in unknown lesions provide a tissue signature that may be used to assess its malignancy.

\section{MATERIALS}

A retrospective analysis of testicular CEUS data from 20 histologically proven testicular lesions (10 seminoma and 10 LCT; size mean: $11.4 \mathrm{~mm}$, range: $3 \mathrm{~mm}-28.6 \mathrm{~mm}$ ) were performed. Ethics approval for reporting was granted from each patient to report this retrospective analysis.

Ultrasound examinations were performed with Acuson Sequoia ${ }^{\mathrm{TM}}$ or a $\mathrm{S} 2000^{\mathrm{TM}}$ system (Siemens Medical Solutions, Mountain View, CA): CEUS data were acquired using harmonic imaging with a low-mechanical index technique (Cadence contrast pulse sequencing (CPS ${ }^{\mathrm{TM}}$ ); Siemens Medical Solutions, Mountain View, CA)) with mechanical index set at or below $0.10 \mathrm{~dB} / \mathrm{cm} / \mathrm{MHz}$, after the administration of a bolus of $4.8 \mathrm{~mL}$ of SonoVue ${ }^{\mathrm{TM}}$ (Bracco $\mathrm{SpA}$, Milan, Italy), a sulfur hexafluoride microbubble contrast agent is injected, followed by $10 \mathrm{~mL}$ of normal saline via an antecubital vein cannula. An imaging plane is fixed and investigated. Continuous observation was usually performed from the time of arrival and the lesion of the microbubbles for at least 90 seconds in most cases (some cases may have reduced periods of observation), using splitscreen mode so that matched CEUS and B-mode frames are available at any time point. 


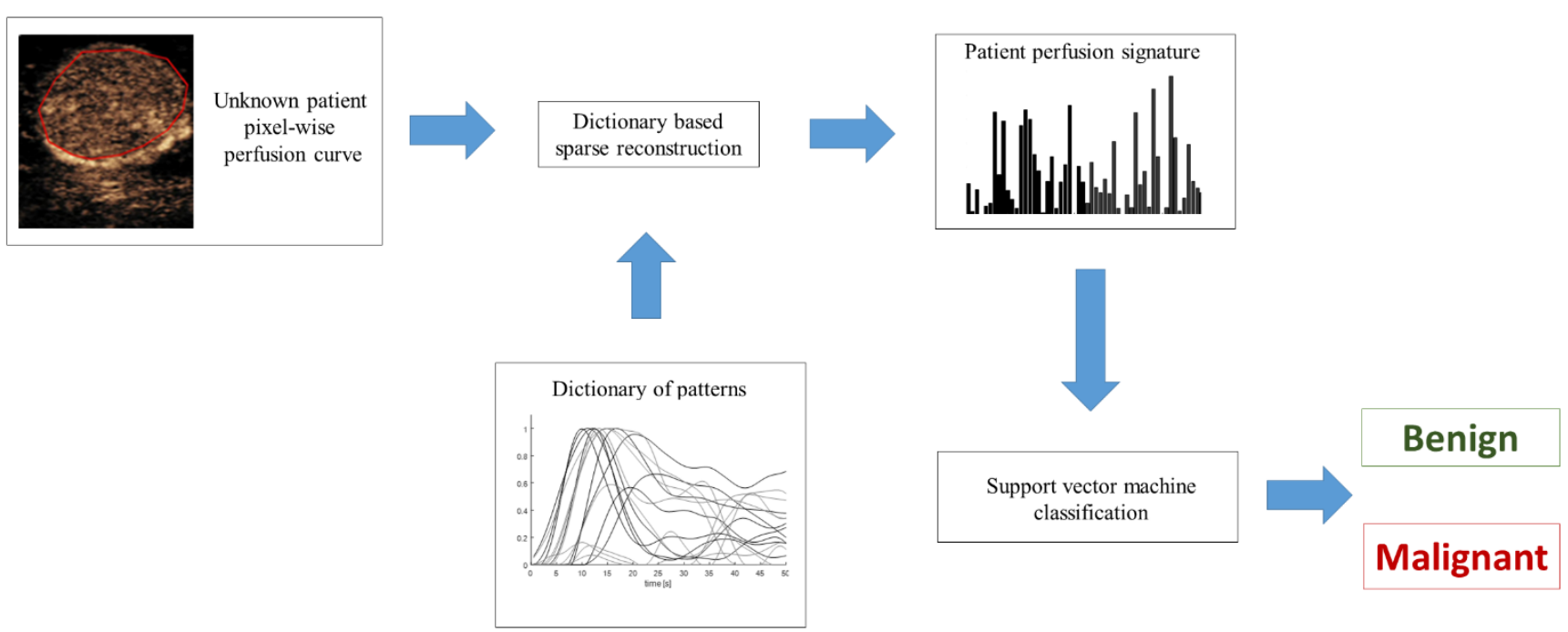

Figure 3 Test phase. The perfusion curves extracted from the CEUS data of a suspect mass with unknown diagnosis are reconstructed using the learnt dictionary, and the resulting signature is fed to the SVM to classify the tissue as benign (LCT) or malignant (seminoma).

\section{METHODS}

\subsection{Preprocessing}

Ultrasound data for each patient underwent the same preprocessing steps, separately.

First, in order to correct for rigid translation of the field of view, a frame by frame registration is applied to the ultrasound data: a user-defined B-mode frame is used as reference, and all other frames are registered to the reference using phase correlation method [15]; the estimated transformation is then applied to all corresponding CEUS frames.

Then, an expert radiologist outlined the region of interest, and all time-intensity curves (TIC) from the pixels within the outlined region are extracted.

In order to account for non-zero baseline signal, this is estimated as the average intensity in the frames before the time of arrival of the contrast and subtracted from the original data [8]. By this means, all pixel-wise TICs start from zero intensity.

Finally, TICs are regularized using a cubic smoothing spline and aligned by shifting in time the curves so to have the same time to peak, set equal to an arbitrary reference value for all patients.

\subsection{Perfusion curve decomposition}

Many real-world data are non-negative and the corresponding hidden components express physical or physiological meanings only when the non-negative condition are imposed [16]. In particular this is especially true when dealing with signals that represent the appearance of a contrast agent within a region. This will also impose than when imaging a discrete region (pixel or voxel), the observed intensity can be only a positive summation of the underlying contribution, coming from possibly heterogenous tissues (partial volume effect). The decomposition of the observed signals into components that are at the same time positive and additive is not ensured by commonly used methods as Singular Value Decomposition, Principal Component Analysis, or Independent Component Analysis. At variance with the aforementioned methods, Nonnegative Matrix Factorization (NMF) imposes the non-negativity constraint on the factorizing matrices so that NMF allows only additive but not subtractive combinations during the factorization and the estimated components are forced to take only positive values. This result in parts-based representation of the data, which can discover hidden components that have specific structures and physical or physiological meanings.

If all TICs extracted from a region of the CEUS data of the $p^{\text {th }}$ patient are stacked into a matrix $Y_{p} \in \mathcal{M}^{N \times T}$, where $N$ is the number of pixels and $T$ is the number of time samples, NMF tries to find a matrix $X_{p} \in \mathcal{M}^{C \times T}$ of $C$ non-negative components of $T$ time samples, that can be added using nonnegative weights $W_{p} \in \mathcal{M}^{N \times C}$ to provide an approximation of the original data $Y_{p}$ :

$$
\underset{X_{p} \geq 0, W_{p} \geq 0}{\operatorname{argmin}}\left\|Y_{p}-W_{p} X_{p}\right\|_{2}^{2}
$$

The number of components is fixed and represent a parameter of the method, so that for each patient $C$ components are extracted.

\subsection{Tissue perfusion signature}

After all $C$ components from all $P$ patients in the dataset are extracted, they are used as words of a dictionary $\mathcal{D}$, that is thus composed of $C \cdot P$ perfusion components. 
When the data from a patient $p$ are available, all $N_{p}$ perfusion curves are stacked in the data matrix $Y_{p}$, and can then be approximated as a non-negative and sparse linear combination of the patterns in the dictionary $\mathcal{D}$. This is achieved by solving the non-negative LASSO optimization:

$$
\underset{A \in \mathbb{R}^{N} p \times C \cdot P}{\operatorname{argmin}}\left\|Y_{p}-A \mathcal{D}\right\|_{2}^{2} \quad \text { s.t. }\left\{\begin{array}{c}
\left\|A_{i,-}\right\|_{1} \leq \mu \\
A_{i,-} \geq 0
\end{array} \forall i=1, \ldots, N\right.
$$

where $A_{i,-}$ is the $i^{\text {th }}$ rows of the coefficient matrix $A \in$ $\mathbb{R}^{N_{p} \times C \cdot P}$. A tissue perfusion signature $\delta_{p} \in \mathbb{R}^{C \cdot P}$ can be obtained using the frequency with which each pattern in $\mathcal{D}$ is used to reconstruct an element in $Y_{p}$. Each $j^{\text {th }}$ element in the signature, with $j=1, \ldots, C P$ can be obtained as:

$$
\mathcal{S}_{p}(j)=\left\|A_{-, j}\right\|_{0} / N_{p}
$$

being $A_{-, j}$ the $j^{\text {th }}$ column of $A$.

\subsection{Classification}

A SVM with a linear kernel has been trained (see Fig. 2 for the entire training pipeline) using the tissue signature $\mathcal{S}_{p}$ from each patient as feature vector, and the corresponding binary diagnosis (malignant seminoma or benign LCT) as target class. When a new patient with a lesion of unknown diagnosis is evaluated, the classification pipeline is exemplified in Fig.3, where the dictionary $\mathcal{D}$ and the SVM classifier is available from the previous training phase.

\section{RESULTS}

The whole procedure (NMF decomposition, dictionary building, SVM training) underwent 10 -fold cross validation. It is worth noting that to ensure a balanced training set, we split the data making sure that in each fold a seminoma and an LCT patient were present.

The number of components has been heuristically fixed to $C=2$ to keep the dictionary size limited and to avoid the decomposition in un-physiological curves, the sparsity parameter in the LASSO optimization has been set to $\mu=$ 0.01 and the penalty parameter C in the SVM is set to 3 .

The resulting test accuracy is $100 \%$.

\section{CONCLUSIONS}

We propose a data-driven pipeline that extract relevant perfusion patterns from the pixel-wise time-intensity curves from contrast-enhanced ultrasound data of lesions with known histology. The presence of this patterns in data from unknown lesions provide a tissue signature that might be used to classify the lesion as benign (LCT) or malignant (seminoma). Despite the perfect classification obtained, it is worth noting the limited number of patient in the cohort, so that the results, although promising, should be validated on larger dataset.

\section{REFERENCES}

[1] D. Y. Huang and P. S. Sidhu, "Focal testicular lesions: colour Doppler ultrasound, contrast-enhanced ultrasound and tissue elastography as adjuvants to the diagnosis," BJR Radiology, vol. 85, no. Spec No 1, pp. S41-5, 2012.

[2] P. Paffenholz et al., "Testis Sparing Surgery for Benign Testicular Masses: Diagnostics and Therapeutic Approaches.," J Urol., vol. 200, no. 2, pp. 353-360, 2018.

[3] P. Albers et al., EAU Guidelines on Testicular Cancer, European Association of Urology, 2016.

[4] N. Nishida et al., "Angiogenesis in Cancer," Vasc Health Risk Manag., vol. 2, no. 3, pp. 213-9, 2006.

[5] T. Nakahara et al., "Effect of inhibition of vascular endothelial growth factor signaling on distribution of extravasated antibodies in tumors," Cancer Research, vol. 66, no. 3, p. 1434-1445, 2006.

[6] H. Alimoradi et al., "Hypoxia Responsive Drug Delivery Systems in Tumor Therapy," Curr Pharm Des., vol. 22, no. 19 , pp. $2808-20,2016$

[7] C. Dudau et al., "Can Contrast-Enhanced Ultrasound Distinguish Malignant from Reactive Lymph Nodes in Patients with Head and Neck Cancers?," Ultrasound in Medicine and Biology., vol. 40, no. 4, pp. 747-754, 2014.

[8] G. Rizzo et al., "Pixel-based approach to assess contrastenhanced ultrasound kinetics parameters for differential diagnosis of rheumatoid arthritis," Journal of Medical Imaging, vol. 2, no. 3, 2015.

[9] G. Rizzo et al., "Bayesian quantification of contrastenhanced ultrasound images with adaptive inclusion of an irreversible component," IEEE Transactions on Medical Imaging, vol. 36, no. 4, pp. 1027 - 1036, 2017.

[10] E. Feleppa, J. Mamou, C. Porter and C. Machi, "Quantitative ultrasound in cancer imaging," Semin. Oncol., vol. 38, no. 1, pp. 136-50, 2011.

[11] J. Hudson et al., "Quantifying Vascular Heterogeneity Using Microbubble Disruption-Replenishment Kinetics in Patients With RenalCell Cancer," Invest Radiol., vol. 49, no. 2, pp. 116-23, 2014.

[12] G. Lock et al., "Early experience with contrast-enhanced ultrasound in the diagnosis of testicular masses: a feasibility study," Urology, vol. 77, no. 5, pp. 1049-53, 2011.

[13] B. Corcioni et al., "Contrast-enhanced ultrasound in the differential diagnosis of small testicular masses," in European Society of Radiology, 2015.

[14] G. Rizzo et al., "Data-driven learning to detect characteristic kinetics in ultrasound images of arthritis," in Clinical Image-Based Procedures. Translational Research in Medical Imaging, Lecture Notes in Computer Science, vol 8680, 2014, pp. 17-24.

[15] B.S. Reddy and B.N. Chattarji, "An fft-based technique for translation, rotation, and scale-invariant image," IEEE Transactions on Image Processing, vol. 5, no. Issue: 8, pp. 1266 - 1271, Aug 1996. 
[16] D.D. Lee and H.S. Seung, "Learning the parts of objects by non-negative matrix factorization," Nature, 401, pp 788-791, 1999. 